\title{
Effect of Pioglitazone on Survival and Renal Function in a Mouse Model of Polycystic Kidney Disease
}

\author{
K.L. Raphael ${ }^{a} \quad$ K.A. Strait ${ }^{a} \quad$ P.K. Stricklett ${ }^{a} \quad$ B.C. Baird ${ }^{a} \quad$ K. Piontek ${ }^{b}$ \\ G.G. Germinob $^{\text {b }}$ D.E. Kohan ${ }^{a}$ \\ a Division of Nephrology, University of Utah Health Sciences Center, Salt Lake City, Utah, and \\ ${ }^{b}$ Division of Nephrology, Johns Hopkins University School of Medicine, Baltimore, Md., USA
}

\section{Key Words}

Polycystic kidney disease $\cdot$ Blood pressure $\cdot$ Pioglitazone

\begin{abstract}
Background/Aims: Cystic epithelia in polycystic kidney disease display features similar to malignant cells. Thiazolidinediones have been shown to have anti-neoplastic properties, therefore we tested the hypothesis that pioglitazone reduces cyst formation, improves renal function, and prolongs survival in a mouse model of polycystic kidney disease. Methods: PC-Pkd1-KO mice, which have homozygous mutations of the $P k d 1$ gene in principal cells, were used. On the day after giving birth, mothers were fed standard mouse chow with or without pioglitazone (30 mg/kg chow). After weaning, the assigned diet was continued. At 1 month of age, blood pressure was measured and animals were sacrificed to determine kidney weight, body weight, and serum urea. Kidneys were evaluated for proliferation using Ki-67, apoptosis using TUNEL analysis, and cyst number using MRI. Survival was observed. Results: Pioglitazone did not alter renal function, cell proliferation, apoptosis, or cyst formation in animals with polycystic kidney disease, however it did increase survival. Pioglitazone reduced blood pressure in PC-
\end{abstract}

Pkd1-KO, but not in controls. Conclusion: These findings suggest that pioglitazone may have a unique antihypertensive effect in polycystic kidney disease, and that such an effect may promote improved survival.

Copyright $\odot 2009$ S. Karger AG, Basel

\section{Introduction}

Autosomal dominant polycystic kidney disease (ADPKD) is the most common heritable cause of end-stage renal disease. There is no specific therapy for ADPKD; progressive renal damage and end-stage renal disease occur in many patients. It is clear that further treatment studies are necessary given the grim prognosis for many ADPKD patients.

Thiazolidinediones (TZDs), synthetic ligands of the peroxisome proliferator-activated receptor- $\gamma$ (PPAR $\gamma$ ), have been shown in human and animal studies to exert beneficial renal effects, particularly in diabetes or obesity. These effects predominantly involved reductions in blood pressure, albuminuria, and inflammation $[1,2]$. Beyond the realm of diabetes, obesity, and renal disease, TZDs are receiving attention in the oncology literature

\section{KARGER}

(C) 2009 S. Karger AG, Basel

Fax +41613061234 E-Mail karger@karger.ch www.karger.com www.karger.com/ajn
Donald E. Kohan, MD, PhD

Division of Nephrology, University of Utah Health Sciences Center

30 North 1900 East

Salt Lake City, UT 84132 (USA)

Tel. +1 801581 6700, Fax +1 801581 4343,E-Mail donald.kohan@hsc.utah.edu 
because of their anti-neoplastic properties. This has been demonstrated in lung, breast, colon, thyroid, melanoma, renal cell, and pancreatic cancer, to name a few. TZDs are anti-neoplastic through: (1) promoting cellular differentiation, (2) inducing apoptosis, (3) being anti-proliferative, (4) inhibiting metastasis, and (5) being anti-angiogenic. Curiously, the anti-neoplastic effects of TZDs are both PPAR- $\gamma$-dependent and independent [3].

Epithelia in ADPKD have features of malignant cells, including disordered apoptosis, de-differentiation, and proliferation [4]. Given their anti-apoptotic and anti-proliferative effects, as well as their benefits on renal function and blood pressure in animal models, we studied the effect of pioglitazone in the PC-Pkd1-KO mouse model of ADPKD. These mice develop cystic kidney disease at 1 week postnatally due to homozygous mutation of the $P k d 1$ gene in principal cells; cystic kidney disease progresses with a mean survival of approximately 2 months [5]. The main objectives of the current study were to determine if pioglitazone: (1) provides a survival benefit, (2) has an effect on renal function, (3) reduces proliferation in cystic epithelia, and (4) reduces cyst formation and kidney weight.

\section{Methods}

\section{Generation of PC-Pkd1-KO Mice}

Principal cell-specific deletion of the $P k d 1$ gene in mice was achieved as previously described [5]. Two lines of mice were used: (1) mice containing a transgene expressing Cre recombinase under the control of the aquaporin-2 promoter (AQP2-Cre) that express Cre selectively in renal collecting duct principal cells, and (2) mice with loxP-flanked exons 1 and 4 of the Pkd1 gene $\left(P k d 1^{c o n d}\right)$. AQP2-Cre transgenic female mice heterozygous for the $P k d 1^{\text {cond }}$ allele were bred with male mice homozygous for the $P k d 1^{\text {cond }}$ allele. Offspring heterozygous for AQP2-Cre and homozygous for the $P k d 1^{\text {cond }}$ allele have principal cell-specific knockout of the Pkd1 gene (PC-Pkd1-KO) and develop cystic kidney disease. Littermates homozygous for the $P k d 1^{\text {cond }}$ allele, but without AQP2-Cre, do not have evidence of cystic kidney disease or renal dysfunction and were used as controls.

\section{Pioglitazone Treatment}

During gestation, all animals received standard mouse chow. On the day after giving birth, mothers were fed standard mouse chow with or without pioglitazone at $30 \mathrm{mg} / \mathrm{kg}$ food. At 3 weeks of age, mice were weaned and genotyped to detect the AQP2-Cre transgene and $P k d 1^{\text {cond }}$ allele. Tail genomic DNA was PCR amplified for the AQP2-Cre transgene using oligonucleotide primers that amplify a region within Cre: CreF (5'-CAT TAC CGG TCG ATG CAA CGA G-3') and CreR (5'-TGC CCC TGT TTC ACT ATC CAG G-3'). DNA was PCR amplified for the $P k d 1^{\text {cond }}$ and $P k d 1^{w t}$ alleles using primers GerF2 (5'-GGC TAT AGG ACA GGG ATG ACA T-3') and GerR6 (5'-CAT ATT CCT CAC CTG GGA
ACA G-3'). The amplified product of the $P k d 1^{\text {cond }}$ allele is 34 base pairs longer than the wild-type allele, corresponding to the loxP site in intron 4 . After weaning, the mice continued on their previously assigned diet.

\section{Survival}

PC-Pkd1-KO and control mice treated with $30 \mathrm{mg} / \mathrm{kg}$ of pioglitazone were allowed to survive until natural death. Their survival was compared to that of untreated PC-Pkd1-KO and control animals.

\section{Renal Function, Kidney and Body Weight}

Treated and untreated PC-Pkd1-KO and control mice were sacrificed at 1 month of age to measure kidney weight, body weight, and serum urea (BioAssay Systems, Hayward, Calif., USA). The blood urea nitrogen (BUN; in $\mathrm{mg} / \mathrm{dl}$ ) was calculated by dividing the urea by 2.14 .

\section{Magnetic Resonance Images}

The number of kidney cysts of treated and untreated PC-Pkd1$\mathrm{KO}$ animals was compared using magnetic resonance images (MRIs). High-resolution MRI experiments were conducted on a 7.0 T scanner (BioSpec 70/30, Bruker BioSpin Co., Billerica, Mass., USA) using a $7.0-\mathrm{cm}$ diameter volume transmit and a $1.0-\mathrm{cm} \mathrm{di-}$ ameter surface receive radiofrequency coils. For each specimen, two sets of $3 \mathrm{D}$ images $(256 \times 128 \times 128$ matrix size at $100 \mu \mathrm{m}$ isotropic spatial resolution) were obtained using standard RARE sequence, including a fluid-attenuated inversion-recovery (600 ms inversion time, $800 \mathrm{~ms}$ TR, $7.3 \mathrm{~ms}$ effective TE, and ETL of 4) image and a proton-density (2,000 ms TR, $7.3 \mathrm{~ms}$ TE, and ETL of 8 ) image. The total scan time for the images was approximately $2 \mathrm{~h}$. Cyst number was totaled from three sagittal sections: one from the center of the kidney; the other two were from either side half way from the center to the edge of the kidney.

\section{Proliferation and Apoptosis}

Paraffin-embedded kidney sections from treated and untreated PC-Pkd1-KO mice were deparaffinized and then subjected to antigen retrieval using $10 \mathrm{~mm}$ sodium citrate at $\mathrm{pH}$ 6.0. Sections were incubated with Ki67 rabbit monoclonal antibody, secondarily incubated with peroxidase conjugated anti-rabbit Ig, developed using 3,3-diaminobenzidine (DAB), and counterstained with hematoxylin (all reagents from Vector Laboratories, Burlingame, Calif., USA). DAB-positive cells were counted in 10 individual sections from each kidney. For apoptosis determination, terminal deoxynucleotidyl transferase dUTP nick end-labeling (TUNEL) staining was performed on kidney sections using a commercially available kit.

Blood Pressure

Blood pressure of treated and untreated PC-Pkd1-KO and control mice was measured by tail cuff (BP-2000, Visitech Systems, Apex, N.C., USA).

\section{Statistics}

Survival analysis was done by the Kaplan-Meier method. Comparisons between pioglitazone-treated and untreated PCPkd1-KO mice and between pioglitazone-treated and untreated control mice were done by two-way analysis of variance. Comparison between cyst number in pioglitazone-treated and un- 


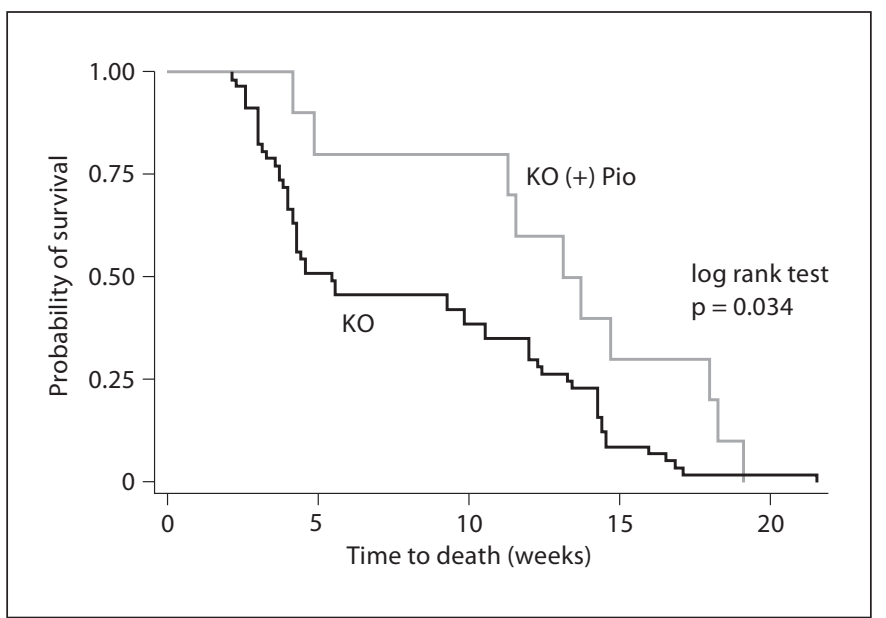

Fig. 1. Kaplan-Meier analysis of survival of pioglitazone-treated or untreated PC-Pkd1-KO animals. PC-Pkd1-KO mice treated with pioglitazone $(\mathrm{n}=10)$ had improved survival compared to untreated PC-Pkd1-KO mice $(\mathrm{n}=57)$.

treated PC-Pkd1-KO mice was done by the Student's t test with unequal variances. $\mathrm{p}<0.05$ was taken as significant. Data are expressed as mean \pm standard error.

Animal Use

All animal experiments were ethically approved by the University of Utah Institutional Animal Care and Use Committee.

\section{Results}

The mean survival of PC-Pkd1-KO animals fed a standard diet was $57.2 \pm 4.9$ days. PC-Pkd1-KO mice fed pioglitazone had a mean survival of $90.2 \pm 11.5$ days; this was longer than untreated PC-Pkd1-KO mice (fig. 1). Control mice, either treated or untreated, were all alive at 3 months after birth.

PC-Pkd1-KO body weight was less than in controls. Pioglitazone increased body weight in control mice $(9.9$ \pm 0.2 vs. $16.3 \pm 0.3 \mathrm{~g})$ and PC-Pkd1-KO mice $(\mathrm{n}=16$, $6.4 \pm 0.5$ vs. $12.5 \pm 0.6$ g; fig. 2). Kidney weight in control mice fed pioglitazone $(228.2 \pm 8.7 \mu \mathrm{g})$ was not different from control mice fed a standard diet $(196.1 \pm 14$ $\mu \mathrm{g})$. However, pioglitazone increased kidney weight in PC-Pkd1-KO mice (759 \pm 55 vs. 1,293 $\pm 206 \mu$ g; fig. 3). Percent body weight attributed to kidney weight was not different in control mice on a standard diet (1.30 \pm $0.03 \%)$ vs. pioglitazone (1.40 $\pm 0.03 \%)$. In PC-Pkd1-KO mice, percent body weight attributed to kidney weight was markedly elevated, however the ratio of kidney:body

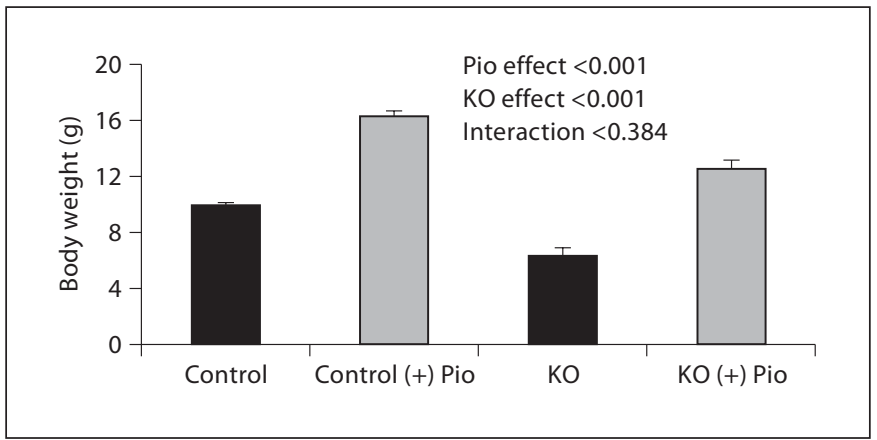

Fig. 2. Effect of pioglitazone on body weight at 1 month of age. Body weight was lower in PC-Pkd1-KO than in control. Pioglitazone increased body weight similarly in control mice $(n=67$ for untreated and $\mathrm{n}=15$ for treated) and PC-Pkd1-KO mice $(\mathrm{n}=16$ for untreated and $n=8$ for treated).

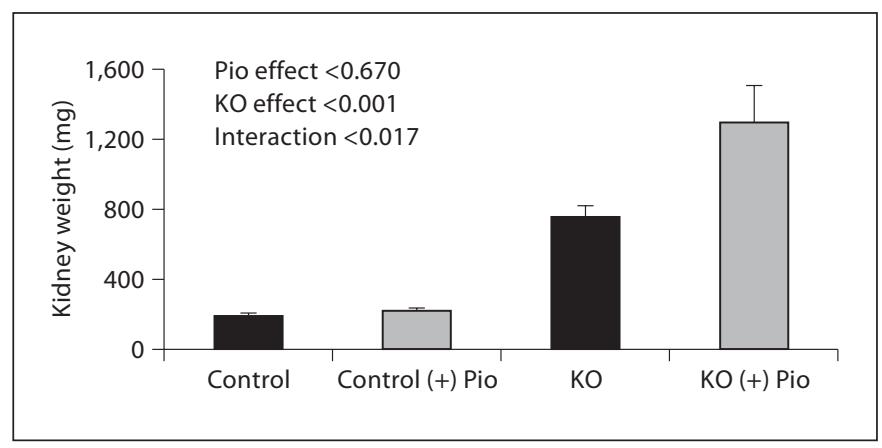

Fig. 3. Effect of pioglitazone on kidney weight at 1 month of age. Kidney weight was greater in PC-Pkd1-KO mice than in controls. Kidney weight in control mice fed pioglitazone $(n=15)$ was not different from control mice fed a standard diet $(n=5)$. Pioglitazone increased kidney weight in PC-Pkd1-KO mice ( $\mathrm{n}=9$ untreated and $n=8$ treated).

weight was similar between PC-Pkd1-KO mice fed a standard diet $(9.1 \pm 1.1 \%)$ or pioglitazone $(10.9 \pm 2.1 \%$; fig. 4).

Pioglitazone lowered BUN in controls (untreated 25.2 \pm 1.3 vs. treated $17.6 \pm 1.0 \mathrm{mg} / \mathrm{dl}$ ) and PC-Pkd1-KO mice (treated $58.9 \pm 2.6 \mathrm{vs}$. untreated $50.3 \pm 5.1 \mathrm{mg} / \mathrm{dl}$ ) when analyzed as a group effect, but there was no difference in the BUN response between the control and PCPkd1-KO groups (fig. 5). PC-Pkd1-KO mice were quite hypertensive. Pioglitazone treatment reduced blood pressure in PC-Pkd1-KO mice $(163.8 \pm 11.7$ vs. $128.2 \pm 3.5$ $\mathrm{mm} \mathrm{Hg}$ ). Blood pressure in treated control mice (109.7 \pm $7.8 \mathrm{~mm} \mathrm{Hg}$ ) was not different from control mice fed a 


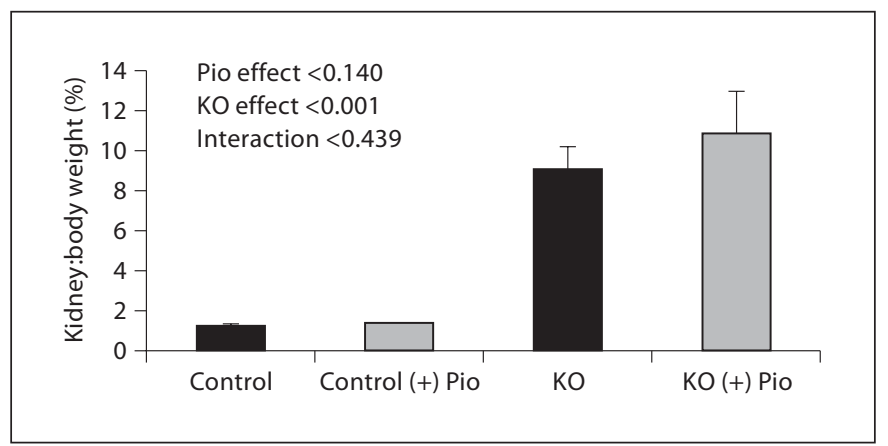

Fig. 4. Percent body weight attributed to kidney weight at 1 month of age. The ratio was not different between control mice on a standard diet $(n=5)$ or pioglitazone $(n=15)$; or between PC-Pkd1-KO mice fed a standard diet $(n=9)$ or pioglitazone $(n=8)$. Kidney: body weight ratio was greater in PC-Pkd1-KO than in controls.

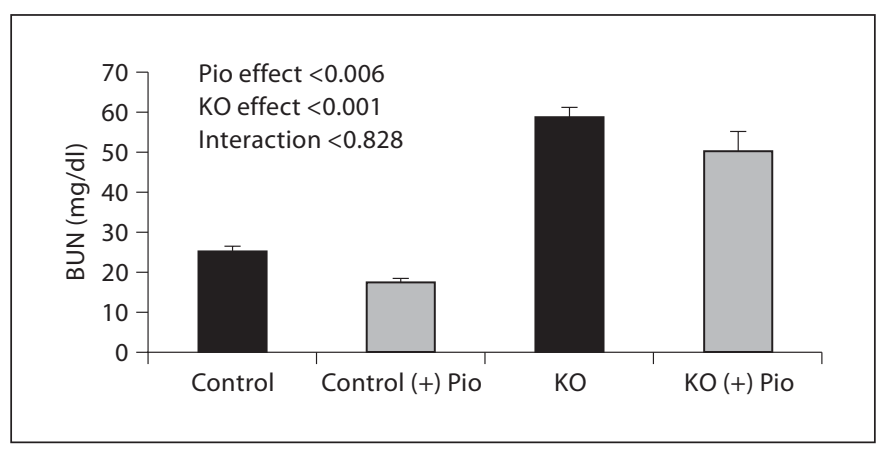

Fig. 5. Effect of pioglitazone on BUN at 1 month of age. BUN was greater in PC-Pkd1-KO than in control mice. Pioglitazone lowered BUN ( $n=15-16$ each group of control mice and $n=8$ each group of PC-Pkd1-KO mice), but this effect was not different between control and PC-Pkd1-KO animals.

standard diet (99.2 $\pm 6.3 \mathrm{~mm} \mathrm{Hg}$; fig. 6). There was no difference in cyst number between PC-Pkd1-KO mice fed a standard diet (166.0 \pm 7.2 cysts/kidney) or pioglitazone (203.4 \pm 31.6 cysts/kidney, $\mathrm{p}=0.294$; fig. 7 ). There was no difference in proliferation between treated and untreated PC-Pkd1-KO mice (fig. 8). Finally, cells undergoing apoptosis were virtually absent in all kidney sections, even in control animals.

\section{Discussion}

The current study demonstrates that mice with polycystic kidney disease live longer if they are treated with pioglitazone. These findings are curious because: (1) PC-

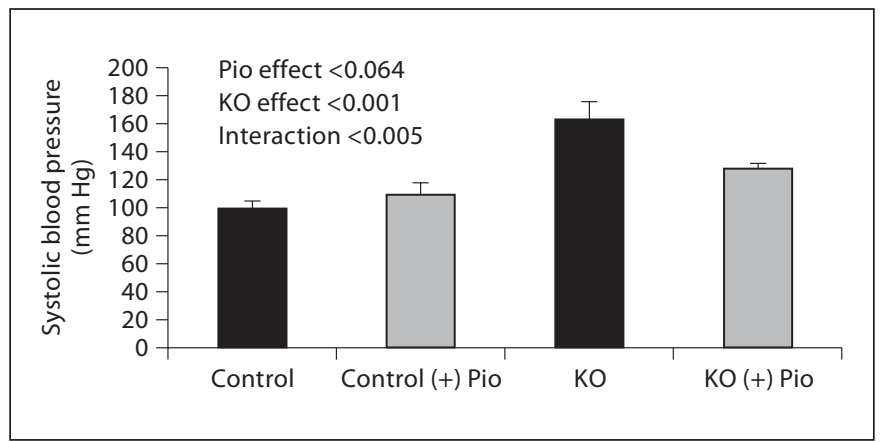

Fig. 6. Effect of pioglitazone on systolic blood pressure at 1 month of age ( $n=5 /$ condition). PC-Pkd1-KO mice were hypertensive. Pioglitazone did not change blood pressure when analyzed as a group effect, however pioglitazone did selectively reduce blood pressure in PC-Pkd1-KO mice when analyzed for interaction.

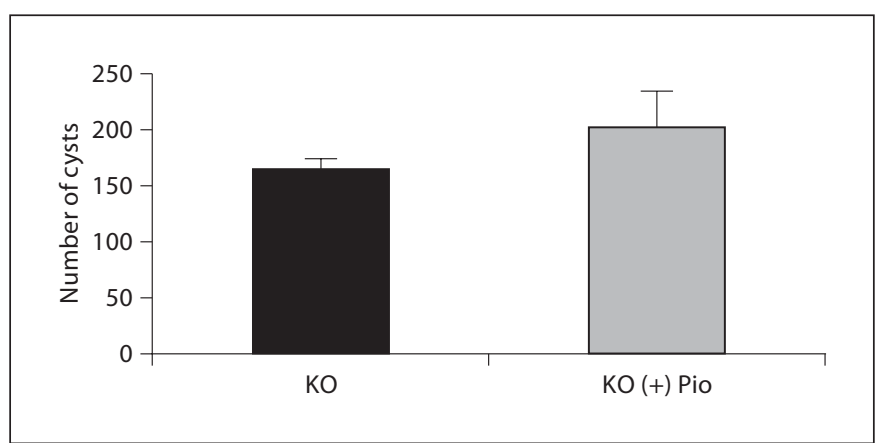

Fig. 7. Effect of pioglitazone on cyst number in PC-Pkd1-KO mice at 1 month of age as assessed by MRI. Cyst number was not changed by treatment ( $\mathrm{n}=8$ /group).

Pkd1-KO mice treated with pioglitazone tended to have more cysts and heavier kidneys than untreated PC-Pkd1 KO mice; (2) the BUN was not different between treated and untreated PC-Pkd1-KO mice, and (3) there was no difference in proliferation or apoptosis. Thus, the current study does not support the notion that pioglitazone reduces cyst formation, cyst enlargement, proliferation or apoptosis. It is possible that pioglitazone reduced fibrosis, however non-cystic tissue is so sparse in the kidney that such a determination is not possible. The only parameter that was significantly improved in PC-Pkd1-KO mice on pioglitazone was blood pressure. Other studies, using pioglitazone in primarily diabetic or obese murine models, have had mixed effects on blood pressure $[1,2]$. Interestingly, in our model, pioglitazone had no effect on blood 
Fig. 8. Effect of pioglitazone on proliferation in PC-Pkd1-KO mice at 1 month of age. Representative images of untreated PC-Pkd1-KO (a) and pioglitazone treated PC-Pkd1-KO mice (b). No difference in proliferation, as assessed by Ki67 staining (brown nuclei), was observed between the groups.
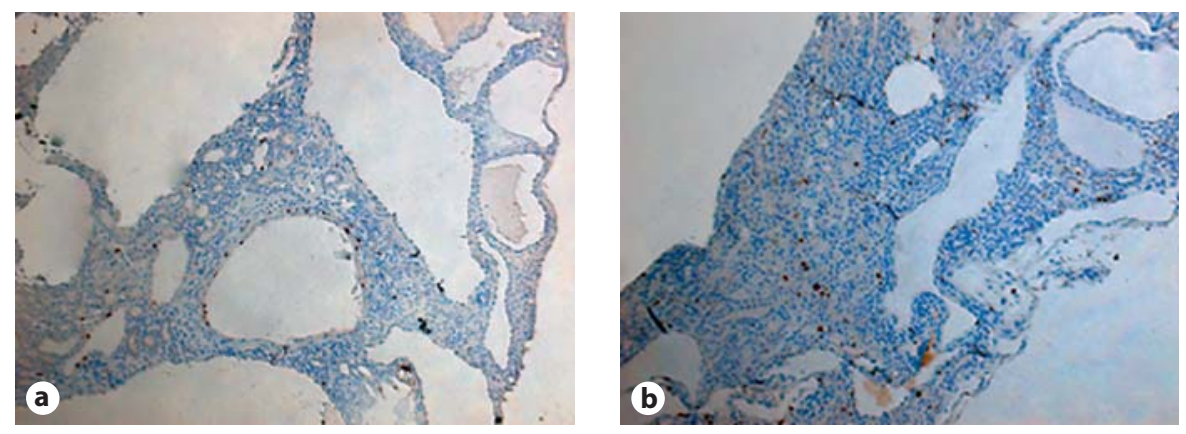

pressure in control mice. The reasons for this are speculative; however, Rodriguez et al. [6] showed that pioglitazone reduced blood pressure in diabetic mice, but not in control mice. While we did not investigate the mechanism(s) responsible for the blood pressure reduction, possibilities include suppression of inflammation and/or improved endothelial function [7-9], conditions that would exist only in the diseased but in not the control animals. Indeed, Muto et al. [10] reported improved endothelial function in heterozygous $P k d 1$ mutants treated with pioglitazone. Regardless of the mechanism(s) involved, the current study suggests that improved blood pressure control in the PC-Pkd1-KO mice led to prolonged survival. That blood pressure reduction has such an effect would not be surprising given the known beneficial result of blood pressure control in improving kidney survival in patients with ADPKD [11]. Finally, it should be noted that the mechanisms by which PPAR $\gamma$ is involved in blood pressure regulation are complex and the subject of ongoing investigation [12]; clarification of the mechanisms involved in the blood pressure reduction seen in PC-PKD1-KO mice are clearly beyond the scope of the current study.

A previous study by Muto et al. [10] demonstrated that pioglitazone reduced cyst area in a $P k d 1$ mutant mouse model. This whole animal $P k d 1 \mathrm{KO}$ model died in utero with cardiac anomalies, fetal hydrops, and renal cysts regardless of pioglitazone treatment. In contrast, PC-Pkd1$\mathrm{KO}$ given pioglitazone did not have a reduction in cyst number. One reason for the difference noted in cyst formation could be related to pioglitazone dose. In the study by Muto et al. [10], a dose of $80 \mathrm{mg} / \mathrm{kg} /$ day was administered to mothers. In the current study, mice were fed pioglitazone dosed at $30 \mathrm{mg} / \mathrm{kg}$ food; assuming a 25 -gram mouse eats $4 \mathrm{~g}$ food/day, this is about $120 \mu \mathrm{g} /$ day or 4.8 $\mathrm{mg} / \mathrm{kg} /$ day of pioglitazone. Perhaps, extremely high doses of pioglitazone reduce cyst formation, however such doses are markedly in excess of what would be expected in humans.

Pioglitazone decreased BUN in both control and PCPkd1-KO mice, while pioglitazone also increased body weight in both groups of animals. These effects are highly likely to be due to fluid retention. Studies by our group in collaboration with others have shown that TZDs induce fluid retention in mice $[13,14]$; these studies localized this effect to activation of the collecting duct epithelial sodium channel leading to enhanced renal sodium retention. In addition to the increase in body weight, kidney weight increased in mice fed pioglitazone. Since cyst number was not affected by drug treatment and since cysts occupy the vast majority of renal tissue, the increase in kidney weight must be due to increased cyst fluid content. Such a finding would not be surprising in light of our findings that pioglitazone induces sodium-retaining mechanisms in the collecting duct principal cell, the cell of origin of cysts in our model.

The cause of death in the PC-Pkd1-KO mice is presumably related to renal failure. Furthermore, the prolonged survival in pioglitazone-treated mice is presumably due to less severe renal dysfunction. Direct demonstration of these assertions is problematic given that BUN (and creatinine, although not measured) is not a highly quantitative marker of renal function in rodents. However, initial studies on PC-Pkd1-KO mice demonstrated that significant PKD1 gene recombination occurred only in the kidney [5], making it apparent that any pathology must originally stem from renal-related issues. The only obvious systemic pathology was hypertension, and this was of a moderate degree that was unlikely to lead to nonrenal end-organ damage. In support of this notion is the absence of gross or histologically apparent abnormalities in non-renal tissues in PC-Pkd1-KO mice in the original study characterizing these animals [5]. Thus, the evidence points to a renal cause of death. It is not possible to 
absolutely rule out other factors, albeit the nature of such factors is not obvious.

There are limitations to this study. These animals may not completely reflect human ADPKD since mice are born with mutations in both $P k d 1$ alleles, while in humans, it is proposed that a mutation of the normal PKD1 allele, or a somatic modifier gene, occurs before the development of renal cysts [15]. Another limitation is that we did not specifically investigate whether the survival benefit conferred by pioglitazone was due to better blood pressure (i.e., using a third treatment group given another antihypertensive agent). However, since poorly controlled blood pressure is already known to increase PKD disease progression [11], such studies were not deemed as being highly informative. Finally, we chose a single dose of pioglitazone. Most studies have used pioglitazone doses between 2.5 and $10 \mathrm{mg} / \mathrm{kg} /$ day $[6-9,16,17]$; we used $5 \mathrm{mg} / \mathrm{kg} /$ day. We did not use the dose given in the study by Muto et al. [10] because those animals received very high doses of pioglitazone for only a few days; our aim was to continue pioglitazone throughout the mouse's life and at tolerable doses.

In conclusion, this study shows that pioglitazone reduces blood pressure in polycystic kidney disease mice. Pioglitazone treatment did not lead to fewer cysts or reduced proliferation. Blood pressure lowering may explain the survival advantage observed in PC-Pkd1-KO mice treated with pioglitazone. No studies have examined the effects of TZDs on renal function in patients with ADPKD; our data suggest that such studies may be warranted.

\section{Acknowledgements}

This research was supported by NIH grants DK96392 (D.E.K.) and DK48006 (G.G.G.); and by AHA grant 0725280Y (K.L.R.). The Bruker BioSpec 70/30 MRI scanner used in the current study was funded in part by a grant (1 S10 RR023017) from NIH NCRR. G.G.G. is the Irving Blum Scholar of the Johns Hopkins University School of Medicine.

\section{References}

1 Sarafidis PA, Bakris GL: Protection of the kidney by thiazolidinediones: an assessment from bench to bedside. Kidney Int 2006; 70 : 1223-1233.

-2 Szeto CC, Li PK: Antiproteinuric and antiinflammatory effects of thiazolidinedione. Nephrology (Carlton) 2008;13:53-57.

-3 Blanquicett C, Roman J, Hart CM: Thiazolidinediones as anti-cancer agents. Cancer Ther 2008;6:25-34.

4 Simons M, Walz G: Polycystic kidney disease: cell division without a c(l)ue? Kidney Int 2006;70:854-864

-5 Raphael KL, Strait KA, Stricklett PK, Miller RL, Nelson RD, Piontek KB, Germino GG, Kohan DE: Inactivation of Pkd1 in principal cells causes a more severe cystic kidney disease than in intercalated cells. Kidney Int 2009;75:626-633

-6 Rodriguez WE, Tyagi N, Joshua IG, Passmore JC, Fleming JT, Falcone JC, Tyagi SC: Pioglitazone mitigates renal glomerular vascular changes in high-fat, high-calorie-induced type 2 diabetes mellitus. Am J Physiol Renal Physiol 2006;291:F694-F701.

-7 Ko GJ, Kang YS, Han SY, Lee MH, Song HK, Han KH, Kim HK, Han JY, Cha DR: Pioglitazone attenuates diabetic nephropathy through an anti-inflammatory mechanism in type 2 diabetic rats. Nephrol Dial Transplant 2008;23:2750-2760.
8 Namikoshi T, Satoh M, Tomita N, Haruna Y, Kobayashi S, Komai N, Sasaki T, Kashihara $\mathrm{N}$ : Pioglitazone ameliorates endothelial dysfunction in obese rats with nephropathy. Biochem Biophys Res Commun 2007;361: 835-840.

9 Omasu F, Oda T, Yamada M, Yoshizawa N, Yamakami K, Sakurai Y, Miura S: Effects of pioglitazone and candesartan on renal fibrosis and the intrarenal plasmin cascade in spontaneously hypercholesterolemic rats. Am J Physiol Renal Physiol 2007;293:F12921298.

10 Muto S, Aiba A, Saito Y, Nakao K, Nakamura K, Tomita K, Kitamura T, Kurabayashi M, Nagai R, Higashihara E, Harris PC, Katsuki M, Horie S: Pioglitazone improves the phenotype and molecular defects of a targeted Pkd1 mutant. Hum Mol Genet 2002;11:17311742.

11 Chapman AB, Guay-Woodford LM, Grantham JJ, Torres VE, Bae KT, Baumgarten DA, Kenney PJ, King BF, Jr., Glockner JF, Wetzel LH, Brummer ME, O'Neill WC, Robbin ML, Bennett WM, Klahr S, Hirschman GH, Kimmel PL, Thompson PA, Miller JP: Renal structure in early autosomal-dominant polycystic kidney disease (ADPKD): the consortium for radiologic imaging studies of polycystic kidney disease (CRISP) cohort. Kidney Int 2003;64:1035-1045.
12 Hamblin M, Chang L, Zhang J, Chen YE: The role of peroxisome proliferator-activated receptor gamma in blood pressure regulation. Curr Hypertens Rep 2009;11:239-245.

13 Guan Y, Hao C, Cha DR, Rao R, Lu W, Kohan DE, Magnuson MA, Redha R, Zhang Y, Breyer MD: Thiazolidinediones expand body fluid volume through PPARgamma stimulation of ENaC-mediated renal salt absorption. Nat Med 2005;11:861-866.

14 Zhang H, Zhang A, Kohan DE, Nelson RD, Gonzalez FJ, Yang T: Collecting duct-specific deletion of peroxisome proliferator-activated receptor gamma blocks thiazolidinedione-induced fluid retention. Proc Natl Acad Sci USA 2005;102:9406-9411.

15 Yoder BK, Mulroy S, Eustace H, Boucher C, Sandford R: Molecular pathogenesis of autosomal dominant polycystic kidney disease. Expert Rev Mol Med 2006;8:1-22.

16 Makino H, Miyamoto Y, Sawai K, Mori K, Mukoyama M, Nakao K, Yoshimasa Y, Suga S: Altered gene expression related to glomerulogenesis and podocyte structure in early diabetic nephropathy of $\mathrm{db} / \mathrm{db}$ mice and its restoration by pioglitazone. Diabetes 2006; 55:2747-2756.

17 Ohtomo S, Izuhara Y, Takizawa S, Yamada N, Kakuta T, van Ypersele de Strihou C, Miyata $\mathrm{T}$ : Thiazolidinediones provide better renoprotection than insulin in an obese, hypertensive type II diabetic rat model. Kidney Int 2007;72:1512-1519. 